Ann. Zootech., I975, 24 (3), 587-593.

\title{
NOTE SUR LA VARIATION TOPOGRAPHIQUE DE L'ÉPAISSEUR DE LA GRAISSE DE COUVERTURE CHEZ LES BOVINS
}

\author{
B. L. DUMONT et G. IROY \\ Laboratoive de Recherches sur la Viande \\ Centre national de Recherches zootechniques, I. N.R. A., \\ 78350 Jouy en Josas
}

\section{RÉSUMÉ}

On a mesuré avec une réglette métallique l'épaisseur de la graisse sous-cutanée en onze sites sur le côté gauche et le côté droit de 69 carcasses de gros bovins de différents types commerciaux (génisses, vaches, bœufs et jeunes taureaux, de races Charollaise, Française Frisonne Pie Noire, Hereford, Limousine, Maine-Anjou, Normande).

La variation de l'épaisseur de graissc a été expliquée de façon significative par le type d'animal et la localisation de la mesure. Les mesures du côté gauche et du côté droit ne différaient pas significativement. On a trouvé de grandes différences entre sites dans leur aptitude à stocker la graisse au fur et à mesure du développement de l'état d'engraissement.

Le relevé de l'épaisseur de gras sous-cutané aux endroits considérés dans cette étude est proposé comme méthode rapide de caractérisation commerciale de la couverture graisseuse des carcasses de gros bovins pour étudier sa variation en fonction des facteurs génétiques ou des conditions d'alimentation.

Dans l'ensemble des tissus adipeux de la carcasse, la graisse de couverture représente, chez les bovins, une part importante qui dépend, notamment, de l'âge, du sexe, clu poids et de l'état d'engraissement (CAllow, I948; Lewis, i967; Johnson, Butterfield et Pryor, 1972).

Ce dépôt graisseux se présente sous la forme d'un revêtement d'épaisseur variable d'une région à l'autre. Cette variation a été utilisée par différents auteurs en vue de déterminer les sites anatomiques où les épaisseurs de gras sont les plus étroitement associées à la composition des carcasses (LINDHÉ, I963; HEDRICK, I968).

Dans leur importance et leur signification les relations entre les épaisseurs de la graisse souscutanée à différents niveaux ont été explorées sur un nombre limité de type zootechnique (LEwis, I967). 
La présente note rapporte les résultats obtenus en étudiant la vatiation de l'épaisseur de gras à divers niveaux de la carcasse sur un lot de bovins de boucherie de différents types commerciaux.

\section{MATÉRIEL, E'T MÉTHODES}

On a considéré soixante-neuf bovins comprenant des sujets des quatre types sexuels (génisse, vache, boufs et mâles entiers) et de différentes races (Charollaise, Française Frisonne Pie Noire, Hereford, Limousine, Maine-Anjou, Normande); les animaux étaient sacrifiés dans nos abattoirs. Vingt-quatre heures après l'abattage, sur chacune des demi-carcasses, refroidie et normalement suspendue par la corde du jarret, on réalisait la mesure de l'épaisseur de la graisse de couverture au niveau des onze sites définis dans le tableau I et représentés à la figure I. Les sites retenus étaient adaptés de ceux proposés par Lindué (I963) et Yentes (I952). Après localisation de l'endroit de la mesure, on procédait, à son niveau, à l'incision de la graisse avec un bistouri. Dans la fente ainsi pratiquée on enfonçait jusqu'à l'appui du muscle sous-jacent une petite réglette métallique graduée en millimètres et munie d'un curseur qu'on laissait affleurer à la limite extérieure de la couche de graisse. En chacun des points définis au tableau I l'épaisseur de gras était mesurée perpendiculairement à la surface de la carcasse. La mesure en $\widehat{I}$ était réalisée en disposant la réglette perpendiculairement à l'axe du sillon séparant les deux muscles Semitendinosus et Semimembranosus.

La figure $I$ indique schématiquement les localisations définies au tableau I .

\section{TABLEAU I}

\section{Description des sites de mesure}

(A) Le point $\mathrm{A}$ est situé latéralement à la carcasse sur une horizontale passant au niveau du bord supérieur de l'apophyse épineuse de la quatrième vertèbre thoracique.

Le point A est à mi-distance du plan médian du corps et de la limite inférieure de la portion dorsale du muscle Trapèze.

(B) Le point B est situé sur l'épine acromienne du Scapulum, au niveau de la tubérosité acromienne.

(C) Le point $\mathrm{C}$ est situé latéralement à la carcasse à $5 \mathrm{~cm}$ du plan médian du corps sur une horizontale passant au niveau du bord supérieur de l'apophyse ćpineuse de la septième vertèbre thoracique.

(D) Le point $\mathrm{D}$ est situé au même niveau que le point $\mathrm{C}$, mais à $10 \mathrm{~cm}$ de côté du plan médian du corps.

(E) Le point $\mathrm{E}$ est situé sur une horizontale passant par le milieu de l'apophyse épineuse de la première vertèbre lombaire, à $5 \mathrm{~cm}$ de côté du plan médian du corps.

(F) Le point $\mathrm{F}$ est situé sur une horizontale passant par le milieu de l'apophyse épineuse de la première vertèbre lombaire, à $15 \mathrm{~cm}$ de côté du plan médian du corps.

(G) Le point $G$ est situé sur une horizontale passant par le milieu de l'apophyse épineuse de la quatrième vertèbre sacrée, à $5 \mathrm{~cm}$ de côté du plan médian du corps.

(H) Le point $\mathrm{H}$ est situé sur une horizontale passant par le milieu de l'apophyse épineuse de la quatrième vertèbre sacrée, à $15 \mathrm{~cm}$ de côté du plan médian du corps.

(I) Le point I est situé dans le sillon séparant les muscles Semitendinosus et Semimembranosus, au niveau de l'horizontale passant par le périnée.

(J) Le point $\mathbf{J}$ est situé sur le muscle Biceps femoris dans la partie médiane du sillon qui sépare les deux portions de ce muscle.

(K) Le point $\mathrm{K}$ est situé au niveau du périnée, dans le plan de section de la carcasse, à l'aplomb de la verticale passant par le bord antéro-inféricur de la symphyse ischio-pubienne. 

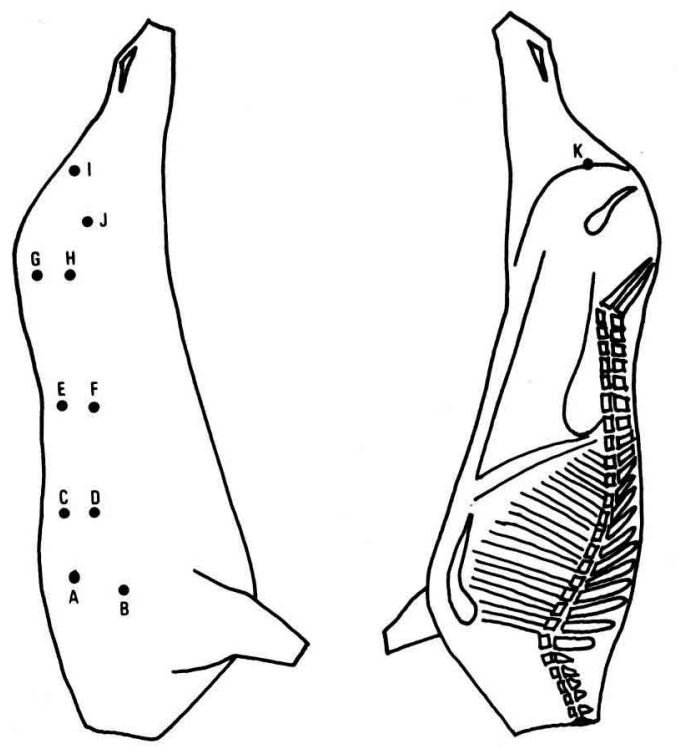

FIG. I. - Localisation des_- sites de mesures

\section{RÉSULTATS ETT DISCUSSION}

Le tableau 2 indique la valeur des moyennes des épaisseurs relevées des deux côtés, ainsi que l'écart type et l'intervalle de variation enregistré.

L'analyse de variance effectuée en considérant comme origines de la variation respectivement la nature des sites anatomiques, le côté mesuré et les animaux, fait apparaître un effet hautement significatif de la nature du site $(F=109,8)$ et des animaux $(F=24,9)$. Il n'existe, par contre, globalement, aucune influence du côté où est effectuée la mesure $(\mathrm{F}=0,05)$.

L'importante variation entre les animaux est logique, puisque les animaux de l'étude avaient été choisis précisément pour représenter un échantillon aussi diversifié que possible des types commerciaux du marché. Or on sait que l'état de gras représente, sur le marché français, un élément variable selon les diverses classes de qualité (Dumont, r967; Dumont et Arnoux, I968).

La variation entre sites est à rapprocher - du moins pour ce qui est des régions dorso-lombaires - des résultats de Lewis (1967); elle amène à considérer qu'aux quatre niveaux de la colonne vertébrale envisagés dans cette étude, de l'avant vers l'arrière, les épaisseurs s'accroissent latéralement à l'axe du corps.

A propos de ce type de mesures on pourrait objecter que le fait de prendre, pour les déterminer, des longueurs fixes $(5 \mathrm{~cm}$, Io $\mathrm{cm}$, ou $15 \mathrm{~cm})$ par rapport au plan médian, conduit à une localisation anatomique variable selon la conformation, le poids de carcasse ct le type d'animal, et que, par suite, les différences d'épaisseurs observées d'un animal à l'autre peuvent être dues à des différences de localisation anatomique des mesures non forcément liées à des différences réelles d'état d'engraissement entre ces animaux. En fait, il ne nous a pas paru possible de déterminer dans ces régions des repères anatomiques d'une autre manière en raison de l'estompement ou de la disparition des zones de repères musculaires dès qu'on se trouve en présence de carcasses manifestant un léger état d'engraissement. 
TABLEAU 2

Épaisseurs de la graisse sous-cutanée aux différents sites (en $\mathrm{mm}$ )

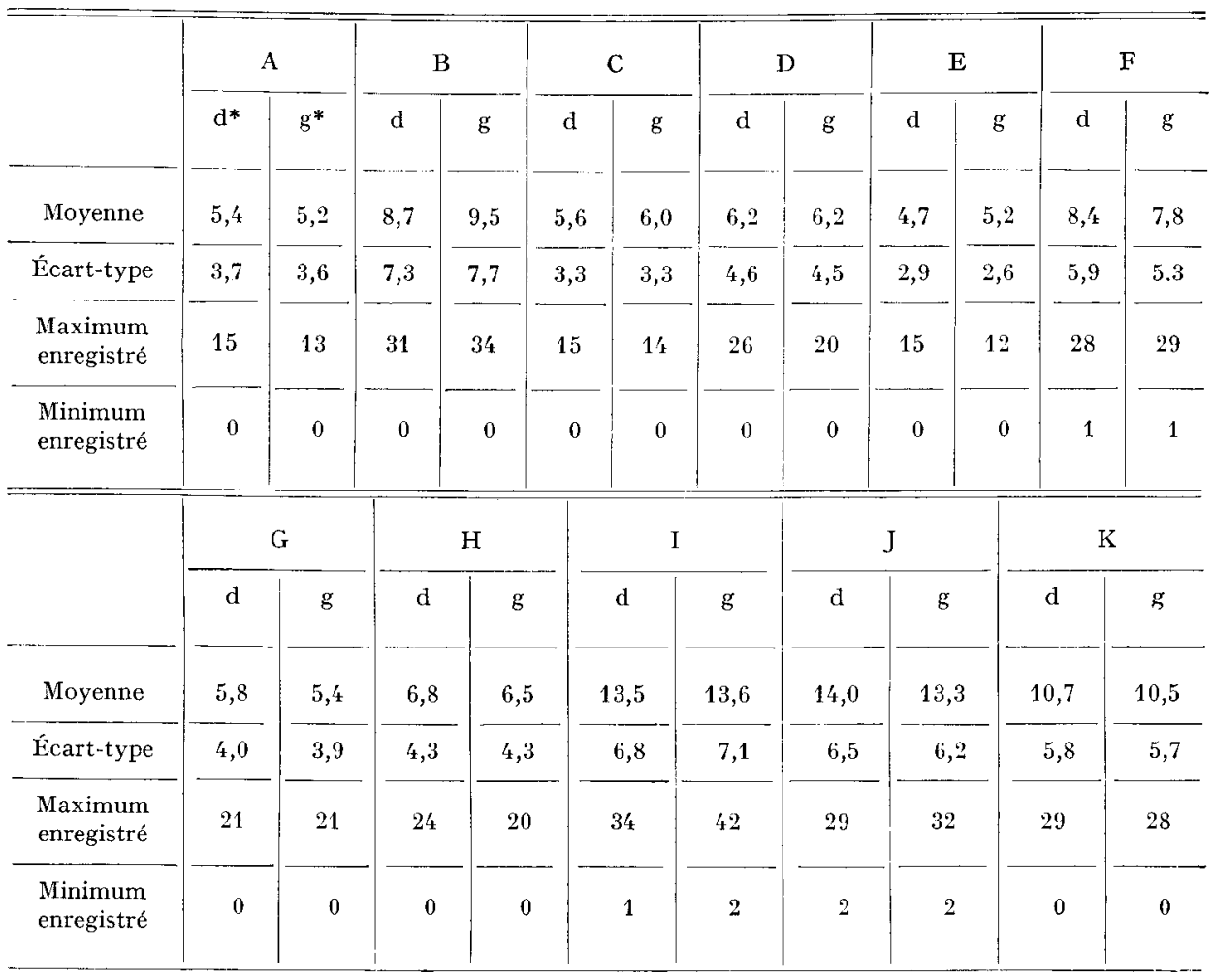

* $\mathrm{d}=$ coté droit ;

$\mathrm{g}=$ côté gauche.

On note aussi, et surtout, que les divers dépôts étudiés correspondent à des zones très différentes dans les capacités de stockage des tissus gras au fur et à mesure que progresse l'état d'engraissement. Le tableau 3 indique les valeurs des coefficients de corrélation entre les épaisseurs de gras aux différents niveaux. D'une façon générale il existe des liaisons positives et significatives entre les valeurs des épaisseurs mesurées dans les diverses zones de la carcasse, mais dans de nombreux cas ces liaisons ne sont pas très étroites, ce qui témoigne d'une relative indépendance des différents sites, les uns par rapport aux autres, en ce qui concerne l'accumulation des graisses de réserve sous-cutanées. Dans le même ordre d'idée on constate des différences sensibles entre sites dans les valeurs des coefficients de variation, et aussi dans l'importance de l'intervalle de variation : chez les animaux les plus maigres de notre échantillon (correspondant à des états de maigreur très prononcée) la presque totalité des sites était dépourvue de graisse, alors que chez les sujets les plus gras, le rapport d'épaisseur entre le site le plus épais et le site le moins épais, était de l'ordre de trois.

A cet égard on doit noter que les trois zones considérées au niveau de la cuisse représentent des dépôts nettement plus importants que ceux du reste de la carcasse. 


\section{TABLEAU 3}

Corrélations entre les épaisseurs des différents sites

\begin{tabular}{|c|c|c|c|c|c|c|c|c|c|c|c|}
\hline & A & B & C & D & $\mathrm{E}$ & F & G & $\mathrm{H}$ & I & $\mathrm{J}$ & $\mathrm{K}$ \\
\hline A & & 0,64 & 0,79 & 0,49 & 0,64 & 0,61 & 0,64 & 0,66 & 0,70 & 0,70 & 0,53 \\
\hline B & & & 0,53 & 0,46 & $0,44^{\prime}$ & 0,41 & 0,51 & 0,42 & 0,62 & 0,73 & 0,54 \\
\hline C & & & & 0,71 & 0,62 & 0,71 & 0,69 & 0,70 & 0,62 & 0,63 & $0,5 / t$ \\
\hline $\mathrm{D}$ & & & & & 0,58 & 0,62 & 0,64 & 0,64 & 0,33 & 0,38 & 0,27 \\
\hline $\mathrm{E}$ & & & & & & 0,66 & 0,64 & 0,72 & 0,54 & 0,62 & 0,35 \\
\hline$F$ & & & & & & & 0,70 & 0,82 & 0,65 & 0,60 & 0,31 \\
\hline G & & & & & & & & 0,75 & 0,56 & 0,65 & $0,,^{\prime} 7$ \\
\hline $\mathrm{H}$ & & & & & & & & & 0,64 & 0,66 & 0,44 \\
\hline I & & & & & & & & & & 0,91 & 0,74 \\
\hline $\mathbf{J}$ & & & & & & & & & & & 0,78 \\
\hline
\end{tabular}

\section{épaisseur de graisse}



FIG. 2. - Profil de la couche de graisse de couverture de trois types commerciaux I : Taurillon Charollais Frison

2: Génisse Limousine

Somme des épaisseurs du côté gauche et du côté droit aux différents sites 
Les observations concernant l'aptitude différentielle des sites au stockage des graisses posent, entre autres problèmes, celui de la pondération à accorder à chacune des mesures dans l'établissement d'équations prédictives de la graisse - graisse totale ou sous-cutanée —à partir d'épaisseurs de tissus gras relevées à différents niveaux. Cette remarque s'applique particulièrement, dans notre esprit, à la relation de LiNDHÉ (I963) qui, considérant la moyenne arithmétique des onze sites nettement différents, accorde donc à chacun d'eux la même importance relative.

Quoi qu'il en soit, d'après l'expérience pratique que nous en avons, le relevé des épaisseurs de gras aux différents niveaux paraît offrir une méthode objective et rapide de description de la répartition simplifiée de la graisse de couverture, utile pour caractériser un type commercial donné. La figure 2 en illustre l'application dans le cas de comparaison de génisses de deux races différentes (Hereford et Limousine) et d'un taurillon Charollais $\times$ Frison.

L'intérêt pratique du rclevé de ces épaisseurs est aussi confirmé par la valeur très élevée $(v=+0,96)$ existant dans l'échantillon considéré ici, entre la somme des épaisseurs et le jugement descriptif détaillé effectué par un expert utilisant une méthode normalisée d'appréciation subjective commerciale (Roy et Dumont, 1975). L'étude détaillée des relations existant entre l'appréciation subjective par cette dernière méthode de la graisse sous-cutanée et les valeurs des épaisseurs de gras aux différents sites de la carcasse est en cours d'élaboration (Dumont et al., 1975).

Un tel relevé définissant un profil de la graisse de couverture pourrait être sans doute utilement employé aussi pour caractériser,"dans les études de la dynamique de l'engraissement, l'influence des divers facteurs susceptibles de le modifier (âge, sexe, poids, race, niveau d'alimentation, etc.). A cette occasion il serait souhaitable de comparer les résultats de ce relevé et ceux de la dissection. Il serait aussi intéressant de comparer les différences d'information apportées par la méthode de routine discutée ici et des méthodes d'exploration systématique de la couverture graisseuse prenant en considération plus de cent sites différents par demi-carcasse (L.EwIS, BRUNGardT et Bray, 1964).

Rę̧u pour publication en janvier 1975.

\section{REMERCIEMENTS}

La réalisation pratique des mesures a été assurée avec l'assistance de M. J. Morand.

Une partie du matériel expérimental nécessaire à ce travail a été fournie par nos collègues de la Station de Génétique quantitative et appliquée (MM. BibE et MENissier). L.es autres animaux ont été choisis avec l'assistance de M. G. Villeneuve. M. J. Lefebvre nous a aidés dans le traitement statistique des données.

Les auteurs sont reconnaissants à ces différentes personnes de l'aide qu'ils ont ainsi apportée à la réalisation de leur projet de travail.

\section{SUMMARY}

\section{SUBCUTANEOUS FAT THICKNESS OF BEEF CARCASSES}

Subcutaneous fat thickness was measured with a steel probe at cleven places (as shown in fig. I) on the left and right sides of sixty-nine beef carcasses from different commercial types (heifers, cows, steers, young bulls, from Charolais, Friesian, Heveford, Limousine, Maine-Anjou and Norman breeds). Significant influences of the type of animal and of locations of measurement were found which explain a major part of the variation of the subcutaneous fat thickness. Measurements on the right and left sides did not differ significantly. Large differences were found between locations for their ability to store fat as fattening proceeds. It is suggested that the contour of subcutaneous fat at the places considered in this study could afford a quick method for characterizing commercially the fat covering of keef carcasses and thus for studying its variation according to breeding or feeding practices. 


\section{RÉFÉRENCES BIBLIOGRAPHIQUES}

CaLlow E. H., 1948. Comparative studies of meat. II. The change in the carcass during growth and fattening and their relation to the composition of the fatty and muscular tissues. J. A gric. Sci., 38, 174-199.

Dumont B. L., 1967. Eitude sur les qualités de carcasses de bovins en France. Informations statistiques, I967 (4), I-72.

Dumont B. L., Arnoux J., I968. Influence de différents caractères de la carcasse de bovins sur la détermination de son prix. Etudes de Statistique agricole, I $968, \mathrm{n}^{\circ} \mathrm{I}$. Office statistique des Communautés européennes.

Dumont B. L., Roy G., Lefebvre J., I975. Étude des correspondances entre deux méthodes d'appréciation de l'état d'engraissement des carcasses de bovins. Ann. Zootech., 24 (4).

Hedrick H. B., ig68. Bovine growth and composition. Mo. Agric. Exp. Sta. Res. Bul, 928.

Johnson E. R., Butterfield R. M., Pryor W. J., ig72. Studies of fat distribution in the bovine carcass. I. The partition of fatty tissues between depots. Aust. J. agric. Res., 23, 38I-388.

Lewis R. W., Brungardt V. H., Bray R. W., I 964 . Contours of bovine subcutaneous fat. I. Influence of weight upon choice grade heifer carcasses. J. anim. Sci. 23, 1203.

LEWIs R. W., I967. Some objective studies related to bef carcass composition. Ph. D. Dissertation University of Wisconsin, Madison-Wisconsin.

LINDHÉ B., I963. Estimation of the relative amount of fatty tissue in beef carcasses by measuring the thickness of the subcutaneous fat. Lantbrukshögsk. Ann., 29, 339-344.

Roy G., Dumont B. L., 1975. Méthode de jugement descriptif de l'état d'engraissement des carcasses de bovins. Revue Méd. vet., 126, 387-40o. 